\title{
Using a Particle Filter to Estimate the Spatial Distribution of the Snowpack Water Equivalent
}

\author{
Philippe CANTET AND M. A. Boucher \\ Département de génie civil et génie du bâtiment, Université de Sherbrooke, Sherbrooke, Quebec, Canada \\ S. LACHANCE-COUTIER AND R. TURCOTTE \\ Direction de l'expertise hydrique, Ministère du Développement durable, de l'Environnement et de la \\ Lutte contre les changements climatiques, Quebec, Quebec, Canada \\ V. FORTIN \\ Centre Météorologique Canadien, Recherche en prévision numérique environnementale, Environnement \\ et Changement climatique Canada, Dorval, Quebec, Canada.
}

(Manuscript received 11 July 2018, in final form 30 January 2019)

\begin{abstract}
A snow model forced by temperature and precipitation is used to simulate the spatial distribution of snow water equivalent (SWE) over a $600000 \mathrm{~km}^{2}$ portion of the province of Quebec, Canada. We propose to improve model simulations by assimilating SWE data from sporadic manual snow surveys with a particle filter. A temporally and spatially correlated perturbation of the meteorological forcing is used to generate the set of particles. The magnitude of the perturbations is fixed objectively. First, the particle filter and direct insertion were both applied on 88 sites for which measured SWE consisted of more or less five values per year over a period of 17 years. The temporal correlation of perturbations enables us to improve the accuracy and the ensemble dispersion of the particle filter, while the spatial correlation leads to a spatial coherence in the particle weights. The spatial estimates of SWE obtained with the particle filter are compared with those obtained through optimal interpolation of the snow survey data, which is the current operational practice in Quebec. Cross-validation results as well as validation against an independent dataset show that the proposed particle filter enables us to improve the spatial distribution of the snow water equivalent compared with optimal interpolation.
\end{abstract}

\section{Introduction}

A spatially accurate assessment of the snow water equivalent (SWE) is thus crucial for streamflow forecasting (e.g., Li and Simonovic 2002) and reservoir level management (e.g., Schaefli et al. 2007) in Nordic areas. Goita et al. (2003) successfully used remote sensing for SWE estimation over the flat and low-vegetated Canadian prairies. However, remotely sensed observations of snow cover can be missing for long periods because of a persisting cloud cover and do not provide a reliable measurement of SWE in forested areas (e.g., Dong et al. 2005), which cover approximately $80 \%$ of the southern portion of the province of Quebec, Canada.

Corresponding author: Philippe Cantet, cantet.philip@gmail.com
A large number of snow models have been proposed in the scientific literature (e.g., Ohmura 2001; Marks and Winstral 2001; Essery et al. 2013). Such models are widely used by operational hydrological forecasting agencies, for instance the National Weather Service River Forecast Centers in the United States (SNOW-17; Anderson 2006) or the Direction de l'Expertise Hydrique du Quebec (HSM; Turcotte et al. 2007).

In Quebec, the most reliable source of SWE observations is the manual snow survey network, which provides roughly one observation every 2 weeks at several sites. However, the date of the measurements can vary greatly from one site to another with delays as long as 3 days. These asynchronous measurements are problematic for data assimilation since precipitation and/or snowmelt is likely to occur between measurements that are part of the same survey campaign. Hence, monitoring 
the spatial and temporal evolution of SWE with interpolation schemes similar to those presented by Seidou et al. (2006) or López-Moreno and Nogués-Bravo (2006) is not possible in that context.

Data assimilation can help to mitigate model deficiencies and uncertainties in the input data to improve the estimation of SWE. The most straightforward and basic data assimilation method is direct insertion, which simply consists of replacing the SWE values simulated by the model with measurements as they become available (e.g., Fletcher et al. 2012). Turcotte et al. (2007) used a spatial interpolation of the model error to correct the estimation of snow density on a $0.1^{\circ}$ grid. Several authors, such as Slater and Clark (2006), recommend against the use of direct insertion methods since they do not account for both model and observation uncertainties and can therefore introduce bias.

Barnett et al. (2005), as well as Liu et al. (2013), improved the snow depth estimations at the continental scale by using optimal interpolation (OI; Burgess and Webster 1980), in which the magnitude of correction depends on the model and observation uncertainties defined a priori. In another study, Magnusson et al. (2014) combined model outputs with point data measurements for SWE using a three-dimensional data assimilation method involving the ensemble Kalman filter (EnKF; Evensen 2003) and OI.

The particle filter $(\mathrm{PF})$ is another data assimilation method that has been successfully used with hydrological models (e.g., Moradkhani et al. 2005a, 2012) and more recently with snow models (e.g., Leisenring and Moradkhani 2011; Magnusson et al. 2017). Unlike EnKF, PFs are robust with regards to nonlinearity and non-Gaussian uncertainty distributions. In addition, since only particle weight is updated, state variables simulated by the model are never modified through assimilation. Rather, they are given a certain level of "credibility" (weight). To the best of our knowledge, as far as SWE is concerned, the recent literature on PF implementation for snow models focuses systematically on applications for a few locations where continuous SWE measurements are available. Thus, the nonsimultaneity of snow survey data over large snow-covered areas has yet to be addressed for data assimilation with the particle filter. In this study, we address this gap and propose a simple methodology to ensure a spatial and temporal coherence of particle weights in the assimilation process. In addition, our method can be used with observations that are irregular in space and time; there is no need to resort to the snow model to evolve states until a common date.

In the following, we first describe the experimental setup, including the study area, model, and data. We then describe our implementation of the particle filter

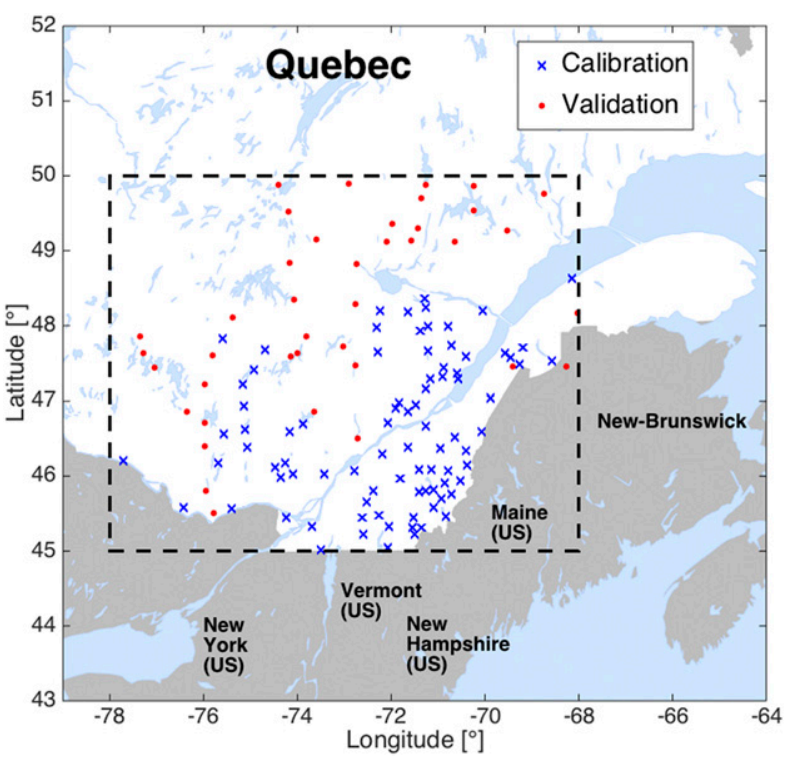

FIG. 1. Localization of the snow survey sites used for the study. The 88 blue " $X$ "s are the sites managed by Quebec's government. The 40 points in red are additional survey points from partner organizations The white area corresponds to the province of Quebec. The study area is the intersection between the dashed rectangle and the white area.

before comparing our results with those obtained by a simple direct insertion method similar to the one in Turcotte et al. (2007). Special care is given to demonstrate the ability of the PF to obtain coherent and reasonably accurate SWE estimations at locations with no observed SWE. This demonstration is performed through leave-one-out cross validation and also through validation with an independent dataset provided by external partners. Finally, we provide a discussion as well as conclusions related to the further improvement of the method.

\section{Experimental setup}

In this section, we describe the study area as well as the available snow survey and climatic data and their characteristics. We then describe the degree-day-based snow model used and its calibration.

\section{a. Study area}

Our study area is the southernmost portion of the province of Quebec, defined here from $68^{\circ}$ to $78^{\circ} \mathrm{W}$ and from $45^{\circ}$ to $50^{\circ} \mathrm{N}$ (see Fig. 1). This area is home to the majority of the province's population and is of high interest for the Direction de l'Expertise Hydrique (DEH), the governmental organization responsible for streamflow forecasting over the public domain. Ground elevation varies from a few meters above mean sea level 
(MSL) near the St. Lawrence River to slightly above $1000 \mathrm{~m}$. According to the observed data within the study area, there are approximately 100 days per year with a maximum temperature under $0^{\circ} \mathrm{C}$ and approximately 150 rainy days, leading to a total annual precipitation of $1100 \mathrm{~mm}$. Of this total, roughly one-third consists of solid precipitation.

\section{b. Available snow survey data}

Figure 1 shows the localization of the 88 observation sites managed by Quebec's government (in blue) as well as an independent dataset (in red) that was made available by partner organizations such as Hydro-Québec, Rio-Tinto, and the Société de Protection des Forêts contre le Feu (SOPFEU). For the remaining, the term "independent dataset" will refer specifically to those points. All of these sites (both blue and red) had some measurements available between fall 2000 and spring 2016. Snow survey sites are characterized by a flat terrain and little exposure to strong winds. The elevation varies from 23 to $858 \mathrm{~m}$ MSL for the blue points and from 91 to $579 \mathrm{~m}$ MSL for the red points. Between four and seven observed values are available per year, depending on the site. Snow survey sites are measured once in January and February, which are typically snow accumulation months, and bimonthly starting in March, which is typically the snow ablation period. Each observation consists of an average of 10 measurements taken manually with a snow tube on a deforested snow path.

From a hydrological perspective, winter starts around the beginning of December and generally ends in April, with an SWE peak at the end of March. The amplitude of this peak varies between 200 and $500 \mathrm{~mm}$, depending on the year and location. Note that measurements are not always available at the exact same date, even for neighboring observation sites. Overall, historical records cover the period between fall 1985 and spring 2000 for 104 observation sites; among those, about 70 correspond to the aforementioned sites.

\section{c. Available climate data}

Daily precipitation and air temperature (minimum and maximum) are available on regular grids with a $0.1^{\circ}$ resolution between 1960 and 2017. These grids were produced through ordinary kriging of ground meteorological stations (Bergeron 2017) operated by the provincial government.

For temperature, a thermal gradient of $0.5^{\circ} \mathrm{C}(100 \mathrm{~m})^{-1}$ of elevation is applied to normalize the temperature at sea level, as in Bergeron (2017). Reciprocally, the same thermal gradient is used to deduce at-site temperature from the normalized temperature and the site's elevation. A spheric variogram is fitted daily for both precipitation and temperature observations, with the maximum distance fixed to $200 \mathrm{~km}$. During the interpolation, only the 10 nearest stations from the target point are used. The interpolation variance is available for both precipitation (generally under $3 \mathrm{~mm}$ ) and temperature $\left(2^{\circ} \mathrm{C}\right)$. In section $3 b(2)$, we explain in greater details how the interpolation variance is exploited in the particle filter implementation.

\section{d. The snow model: HYDROTEL snowpack model}

The snow model, named HYDROTEL Snowpack Model (HSM), used in this study is the same as in Turcotte et al. (2007), to which the reader can refer for greater details. HSM is a temperature-index model part of a broader hydrological model named HYDROTEL (Fortin et al. 2001) that emulates a simplified energy balance. The model only requires temperature and precipitation as meteorological forcing and has five state variables, namely, SWE, heat deficit, albedo, snow depth, and liquid water retention within the snow cover. The main physical processes within a vertical snow column are represented (albeit simplified) in the model and the corresponding equations are dependent on six parameters that require iterative calibration (see Table 1). Note that, in this study, a multiplicative factor parameter for precipitation (pfc) was added.

Each simulation can be decomposed into three steps. First, at each time step, the phase of precipitation (rain or snow) is determined based on air temperature. Solid precipitation is added to the snow cover, and the corresponding effect on the energy budget of the snowpack is evaluated from the specific heat of the snow. If precipitation is liquid, this water is added to the total snow water equivalent, and the heat deficit is adjusted. Second, at each time step, the heat provided at the surface of the soil allows for a decrease in the heat deficit. In HSM, the external energy balance originating from solar radiation is approximated based on the temperature index. If the energy balance of the snow cover is estimated to be positive, snowmelt will occur. If the meltwater volume is greater than the retention capacity of the snowpack, the snow cover retains only the amount of water equivalent to the retention capacity. The rest is considered as runoff at the snow-soil interface and thus leaves the snowpack.

\section{e. Calibration of the snow model HSM}

In this study, the parameters of the HSM model are globally calibrated using the SCE-UA algorithm (Duan et al. 1992). Here, "globally" means that all measurement sites have been concatenated in a single series to estimate the value of the objective function, which here is the NashSutcliffe efficiency index (NSEg; Nash and Sutcliffe 1970) 
TABLE 1. Bounds of parameters and their optimized values using SCE-UA.

\begin{tabular}{|c|c|c|c|c|}
\hline Parameter & Definition & Unit & Bounds & Value from SCE-UA \\
\hline $\mathrm{MR}_{s-s}$ & $\begin{array}{l}\text { Melt rate coefficient at the soil-snow } \\
\text { interface }\end{array}$ & $\mathrm{m} \mathrm{day}^{-1}$ & {$\left[10^{-4}, 10^{-3}\right]$} & $5.46 \times 10^{-4}$ \\
\hline $\mathrm{MR}_{a-s}$ & $\begin{array}{l}\text { Melt rate coefficient at the air-snow } \\
\text { interface }\end{array}$ & $\mathrm{m} \mathrm{day}^{-1}{ }^{\circ} \mathrm{C}^{-1}$ & {$\left[10^{-4}, 10^{-3}\right]$} & $3.53 \times 10^{-3}$ \\
\hline$T_{0}$ & $\begin{array}{l}\text { Temperature threshold at which melting } \\
\text { occurs }\end{array}$ & ${ }^{\circ} \mathrm{C}$ & {$[-3,1]$} & 0.54 \\
\hline$\rho_{\max }$ & Upper bound for density & $\mathrm{kg} \mathrm{m}^{-3}$ & Fixed & $800-8.53 \times$ lat \\
\hline$C_{c}$ & Compaction coefficient & day $^{-1}$ & {$\left[10^{-3}, 10^{-1}\right]$} & $5.4 \times 10^{-3}$ \\
\hline$T_{l-s}$ & $\begin{array}{l}\text { Temperature above which precipitation } \\
\text { is rain }\end{array}$ & ${ }^{\circ} \mathrm{C}$ & {$[-3,1]$} & -0.34 \\
\hline pfc & Precipitation factor correction & - & {$[0.5,1.5]$} & 0.92 \\
\hline Score in Open Loop & & Unit & Validation sites & Calibration sites \\
\hline$N_{S}$ & Number of observation sites & - & 40 & 88 \\
\hline$N_{\text {obs }}$ & Number of observations & - & 2732 & 7522 \\
\hline NSEg & Global Nash-Sutcliffe efficiency index & - & 0.56 & 0.69 \\
\hline NSEl & Local Nash-Sutcliffe efficiency index (IC95\%) & & {$[-0.62,0.89]$} & {$[-1.14,0.87]$} \\
\hline RMSE & Root-mean-square error & $\mathrm{mm}$ & 57.8 & 57.9 \\
\hline MBE & Mean bias error & $\mathrm{mm}$ & -29.3 & 8.7 \\
\hline
\end{tabular}

(defined in Table 2). Note that, for the objective function, the NSEg is computed from the square root of simulated and observed values. This enables us to decrease the weight of very large values in the calculation of NSEg. Hence, this means that the parameters in HSM are not fine-tuned separately for each site, but are rather akin to compromise values that allow for a globally good performance over the entire study domain. Of course, those parameters are a better fit for some sites than for others. Only the $\rho_{\max }$ parameter is fixed and is related to the latitude (according to observation).

The calibration of the snow model has been performed using snow survey data from the calibration sites (blue points on Fig. 1) between 2000 and 2016. This corresponds to 8447 observed point values for
SWE. The value of the calibrated parameters, their bounds, and the corresponding performance scores for the calibration and validation sites are summarized in Table 1 . Performances in validation are not as good as in calibration, which can be explained by the fact that the calibration and validation sites are concentrated in different geographic areas. For instance, the validation dataset includes several sites from Hydro-Québec, a hydropower producer whose operations are mostly located in the north of Quebec. In particular, we observe a negative bias [mean bias error (MBE)] for the validation sites.

In general, the snow model HSM shows reasonable skill in simulating the SWE (see Table 1). Indeed, the NSEg is approximately 0.7 for the calibration period and 0.65 for the validation period (1985-2000) while the

TABLE 2. Summary of performance metrics used in this study.

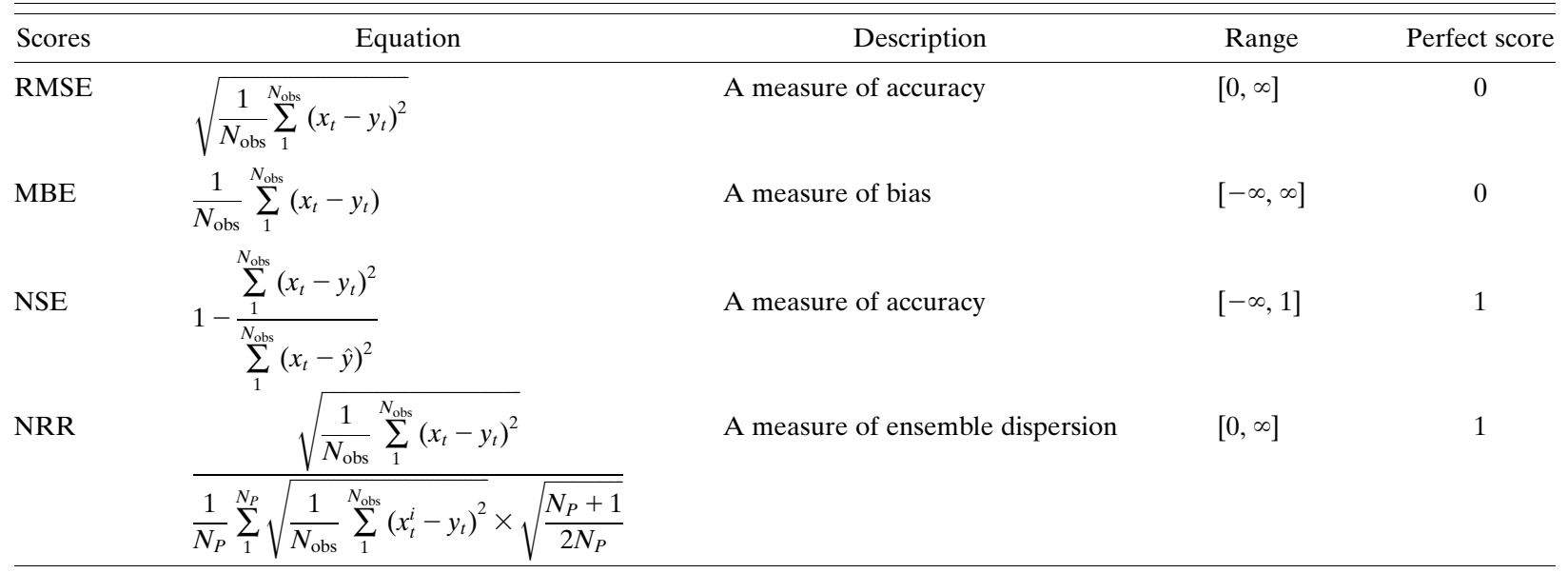


RMSE is respectively 57.9 and $58.2 \mathrm{~mm}$. The RMSE increases during the snow season: for example, the RMSE for January is about $41 \mathrm{~mm}$ whereas in April it is about $65 \mathrm{~mm}$. Table 1 shows that the model slightly overestimates SWE over the calibration period, with an MBE of $8.7 \mathrm{~mm}$

Even though the calibration was performed globally, at-site performances were still examined. Ten percent of sites have a negative local NSE (NSEl) and an RMSE superior to $80 \mathrm{~mm}$, which correspond to a very poor simulation of SWE. Nevertheless, for $50 \%$ of sites, model performance can be considered satisfactory and even good for some sites, since the NSEl exceeds 0.55 and the RMSE does not exceed $50 \mathrm{~mm}$. In addition, it appears that there are no relationships between model performance and variables such as altitude, latitude, and the kriging variance of meteorological data. Only the MBE seems to be linked with altitude. It appears normally distributed, centered on $15 \mathrm{~mm}$, and values do not exceed $\pm 90 \mathrm{~mm}$.

Note that the dual state-parameter framework is a good alternative for calibrating a hydrologic model and was successfully used in low- to high-dimension problems (e.g., Dechant and Moradkhani 2011; Zhang et al. 2017; Abbaszadeh et al. 2018). However, it is not easily applicable in this paper since the snow model needs to be calibrated everywhere, even at locations with no observed SWE (also see section 5 for more details).

\section{f. Performance metrics}

The metrics used to evaluate the performance of each method proposed in this study are defined in Table 2. Note that the Nash-Sutcliffe efficiency index is computed using the median of the ensemble prediction. The ensemble dispersion is evaluated by the normalized rootmean-square error ratio (NRR). A value greater than 1 indicates the ensemble has too little spread relative to the predicted mean and a value less than 1 has too much spread (Moradkhani et al. 2005b).

Let $N_{\text {obs }}$ be the number of observed SWE at a given site and $N_{P}$ be the number of particles. Let $y_{t}$ be the observed SWE at time $t$ and $\hat{y}$ be the mean of $\left\{y_{t}\right\}_{t=1, \ldots, N_{\text {obs }}}$. Let $x_{t}^{i}$ be the predicted value for the particle $i$ at time $t$ and $x_{t}$ be the median of the ensemble prediction $\left\{x_{t}^{i}\right\}_{i=1, \ldots, N_{P}}$ at time $t$.

The next section provides a recapitulation of the general principles guiding the implementation of the particle filter as well as a more in-depth description of our specific implementation.

\section{Data assimilation with the particle filter}

We first provide a short description of the particle filter, before explaining how it is applied specifically in this study. Then, we propose a method to apply the particle filter at unobserved locations over a large territory.

\section{a. General description}

The particle filter originates from the sequential Bayesian framework, which is a probabilistic approach for evolving the state variables of a dynamical system. It consists of two steps.

The first step is called "prediction" and aims at evolving the process. This evolution is necessarily defined by the transitional probability of the model process $p\left(x_{t} \mid x_{1: t-1}\right)$. If the dynamical system can be assumed to follow a Markovian process, then $p\left(x_{t} \mid x_{1: t-1}\right)=p\left(x_{t} \mid x_{t-1}\right)$ and we can write

$$
\mathbf{x}_{t}=f\left(x_{t-1}, u_{t}, \theta_{t}\right)+\omega_{t},
$$

where $\mathbf{x}$ is the state vector, $f($.$) is the state function, \mathbf{u}$ is a vector containing the model inputs, $\boldsymbol{\theta}$ contains the model parameters, $\omega$ is the process noise, and $t$ denotes the time index.

The second step, called "updating," uses new available observations in order to estimate the posterior distribution $p\left(x_{t} \mid y_{1: t}\right)$, which is the conditional distribution of the current state given all available observations. The relationship between $\mathbf{x}_{t}$, the state of the system at time $t$, and the observation, noted $y_{t}$, can be written as

$$
y_{t}=h\left(\mathbf{x}_{t}\right)+\varepsilon_{t},
$$

where $h$ is the observation operator, and $\varepsilon$ is the measurement noise.

Suppose that the posterior distribution is known at $t-1$. Then, the prior probability density function at time $t$ can be computed as follows:

$$
p\left(\mathbf{x}_{t} \mid y_{1: t-1}\right)=\int p\left(\mathbf{x}_{t} \mid \mathbf{x}_{t-1}\right) p\left(\mathbf{x}_{t-1} \mid y_{1: t-1}\right) d \mathbf{x}_{t-1} .
$$

Bayes's law enables us to update this prior density using the observation $y_{t}$, according to

$$
p\left(\mathbf{x}_{t} \mid y_{1: t}\right) \propto p\left(y_{t} \mid \mathbf{x}_{t}\right) p\left(\mathbf{x}_{t} \mid y_{1: t-1}\right)=\frac{p\left(y_{t} \mid \mathbf{x}_{t}\right) p\left(\mathbf{x}_{t} \mid y_{1: t-1}\right)}{p\left(y_{t} \mid y_{1: t-1}\right)} .
$$

The normalization constant is given by

$$
p\left(y_{t} \mid y_{1: t-1}\right)=\int p\left(y_{t} \mid \mathbf{x}_{t}\right) p\left(\mathbf{x}_{t} \mid y_{1: t-1}\right) d \mathbf{x}_{t} .
$$

In general, this problem cannot be solved analytically. Consequently, the posterior filter density is approximated by using an ensemble of model simulations called "particles." Each particle is obtained by running the 
simulation model with either randomly perturbed state variables, randomly perturbed input variables, or both. Hence, if $N_{P}$ particles are used, the posterior filter density is approximated by

$p\left(\mathbf{x}_{t} \mid y_{1: t}\right) \approx \sum_{i=1}^{N_{P}} w_{t}^{i} \delta\left(\mathbf{x}_{t}-\mathbf{x}_{t}^{i}\right), \quad$ where $\quad \sum_{i=1}^{N_{P}} w_{t}^{i}=1$.

At time step $t$, the $i$ th particle is characterized by its position $x_{t}^{i}$ and its weight $w_{t}^{i}$. The latter is recursively computed as follows:

$$
w_{t}^{i}=\frac{w_{t-1}^{i} p\left(y_{t} \mid \mathbf{x}_{t}^{i}\right)}{\sum_{i=1}^{N_{P}} w_{t-1}^{i} p\left(y_{t} \mid \mathbf{x}_{t}^{i}\right)},
$$

where $p\left(y_{t} \mid \mathbf{x}_{t}^{i}\right)$ is the likelihood function and corresponds to the probability density function of the measurement uncertainty [see section $3 \mathrm{~b}(1)$ ].

After a few iterations of the two steps described above, it often happens that the weights of all particles but one become very small or null. This is called filter degeneracy and must be avoided. A simple mean to avoid degeneracy is to resample particles if the effective sample size, given by Eq. (8), falls below a given threshold:

$$
N_{t}^{\mathrm{eff}}=\frac{1}{\sum_{i=1}^{N_{P}}\left(w_{t}^{i}\right)^{2}} .
$$

\section{b. The implementation of the particle filter in this study}

In Eqs. (1) and (2), $f($.$) is the HSM snow model$ described in section $2 \mathrm{~d}$ and $\theta$ is the set of calibrated parameters (see section 2e). Parameter $u_{t}$ is the meteorological data at time $t$ (see section $2 \mathrm{c}$ ), $\mathbf{x}_{t}$ is a vector of the state's variables of the model, and $y_{t}$ is the observed SWE at time $t$. Parameter $h$ is the observation operator, meaning that $h\left(\mathbf{x}_{t}\right)$ provides the SWE estimated by the snow model at time $t$.

Note that, in order to simplify notations, we consider in the rest of the paper that $\mathbf{x}_{t}$ is a scalar which corresponds to the SWE estimated by the snow model at time $t$. Consequently, the function $h$ reduces to the identity function.

Thus, it remains to define the measurement noise $\varepsilon_{t}$, the process noise $\omega_{t}$, and the resampling procedure to implement the particle filter.

\section{1) MeAsurement NOISE: LiKelihood FUNCTION}

Measurement noise, characterized by $\varepsilon_{t}$ in Eq. (2), is needed to define the likelihood function when calculating the particle weights during the updating step [Eq. (7)]. Measurement noise relates to the uncertainty of SWE measurements. In our case, measurements refer to SWE values obtained by manual snow survey. The uncertainty of these measurements is assumed to be fairly small since each observation consists in an average of 10 measurements (Lopez-Moreno et al. 2011). There is, however, a relatively large spatial representativeness error related to these local measurements. The snow cover is known to exhibit quite a large spatial variability. This error must be accounted for in the distribution of measurement noise.

We assume that both types of uncertainty (measurement and spatial representativeness error) are represented by a heteroscedastic Gaussian process of the form:

$$
y_{t} \mid \mathbf{x}_{t}^{i} \sim \mathscr{N}\left(y_{t}-\mathbf{x}_{t}^{i}, \sigma^{2}\right), \text { where } \sigma=0.2 y_{t}+2 .
$$

\section{2) Process NOISE: Generating PARTICles}

A model is ever only a simplified version of reality that rests on a series of assumptions. In particle filtering, the process noise, characterized by $\omega_{t}$ in Eq. (1), allows us to account for model uncertainty. In this way, it is common to apply random perturbations to the state variables $\mathbf{x}_{t}$, to account for the fact that the "true" state variables might not be exactly those simulated by the model. Here, we choose to perturb the predicted SWE by the multiplicative uniform perturbation of Clark et al. (2008) and also used in Leisenring and Moradkhani (2011). In addition, the process noise must also account for the uncertainty related to the observation of meteorological variables which are used as inputs for the simulation model HSM. Indeed, temperature and precipitation are subject to uncertainties because of measurement itself, but mostly due to their spatial interpolation (see section 2c). Accordingly, meteorological input data were perturbed using an additive noise that follows a Gaussian distribution in which the variance is the kriging variance. This choice was motivated by a desire to consider perturbations that are as objective and close to the "real" uncertainty of meteorological observations.

Thus, the prediction step [Eq. (1)] can be rewritten as follows:

$$
\mathbf{x}_{t}^{i}=f\left(\mathbf{x}_{t-1}^{i}, \hat{u}_{t}^{i}, \theta\right) v_{t}^{i}
$$

where $\hat{u}_{t}^{i}$ represents perturbed input data at time $t$ and $v_{t}^{i} \sim \mathscr{C}\left[1-\gamma_{S}, 1+\gamma_{S}\right]$. The value of $\gamma_{S}$ was tuned and fixed to 0.02 especially to control the dispersion of the ensemble (through the NRR index): increasing $\gamma_{S}$ also increases the spread of the ensemble. In fact, the accuracy of the PF is not sensitive to $\gamma_{S}$, except if it exceeds 0.05 . 

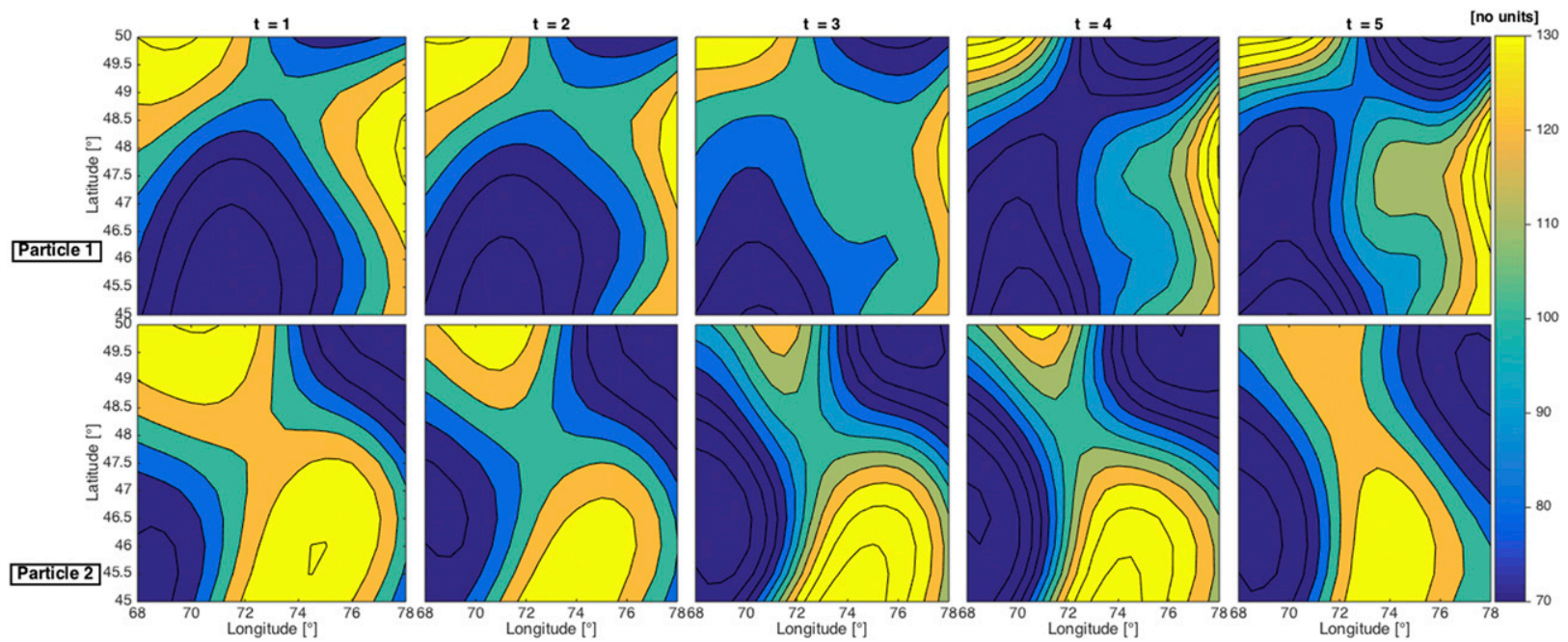

FIG. 2. Illustration of temporally and spatially correlated perturbations on a grid equivalent to the study area: two particles independently perturb an initial value of 100 with a multiplicative noise following $\mathscr{C}[0.7,1.3]$ during five time steps. The time correlation is fixed to $\alpha=0.95$ and the horizontal correlation length scale to $L=300 \mathrm{~km}$ in Eqs. (11) and (13).

\section{3) MAINTAINING COHERENT TEMPORAL AND SPATIAL CORRELATIONS FOR PERTURBATIONS}

It is also important to consider a temporal correlation in the process noise since our modeling framework involves working with meteorological data. For instance, Evensen (1994) proposed to generate the random noise $s_{t}$ recursively as follows:

$$
s_{t}=\alpha s_{t-1}+\sqrt{1-\alpha^{2}} \eta_{t}, \quad \text { with } \quad s_{0} \sim \mathscr{N}(0,1),
$$

where $\eta_{t}$ is a white noise with zero mean and a variance equal to 1 . Parameter $\alpha$ determines the time correlation and can be estimated as

$$
\alpha=1-\frac{\Delta t}{\tau},
$$

where $\Delta t$ is the model time step and $\tau$ is the length scale of time correlation. It is then trivial to transform the normally distributed noise $s_{t}$ into any specified distribution. Parameters $\tau$ and $\alpha$ were tuned and set respectively to 20 days and $\alpha=0.95$. Like for $\gamma_{S}$, the accuracy (and bias) of the PF is not noticeably affected by the value of $\tau$, except if it becomes less than 3 days. There is still a link between $\tau$ and the ensemble spread. A greater value for $\tau$ increases the ensemble spread.

In our implementation, we also consider a spatial correlation in the process noise. We adopted the structure suggested by (Uboldi et al. 2008):

$$
r=\exp \left(\frac{-d^{2}}{L^{2}}\right)
$$

where $r$ is the correlation between two points, $d$ is the horizontal distance, and $L$ is the length scale of the horizontal correlation. This choice of length scale for horizontal correlation has no impact on the performances of the local PF (accuracy, bias, and ensemble dispersion), but helps maintaining the spatial coherence of the particle weights (see next subsection). Parameter $L$ was set to $300 \mathrm{~km}$ because preliminary tests showed that this value allows for the range of the variogram to be larger than with smaller values. However, the same tests have also shown that an $L$ larger than $300 \mathrm{~km}$ did not further increase the variogram range. Since a large range implies that spatial dependency between grid points is maintained for larger spatial scales, this is an interesting property to preserve in our context.

Figure 2 shows the temporal and spatial structure, which can be modeled by Eqs. (11) and (13), respectively. It illustrates how an initial value of 100 is perturbed twice using two independent particles with a multiplicative noise following $\mathscr{C}[0.7,1.3]$ during five time steps. The time correlation is fixed to $\alpha=0.95$ [in Eq. (11)] and the horizontal correlation length scale to $L=300 \mathrm{~km}$ [in Eq. (13)]. The same dependency structure is used to perturb meteorological data (model inputs) and predicted SWE.

By construction, kriging variance varies in space and time. However, this spatiotemporal structure is not necessarily in agreement with the one in Eqs. (11) and (13). Consequently, the use of the kriging variance could affect the spatiotemporal structure that we aim at implementing. To verify that, Fig. 3 compares the temporal and spatial correlation of perturbations when 
(a)

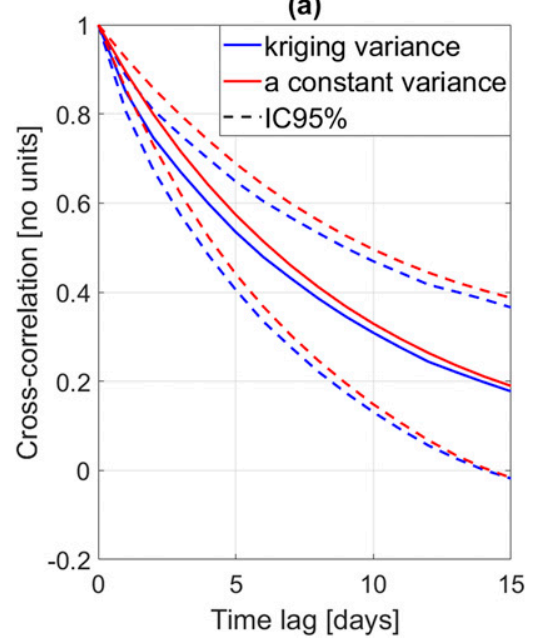

(b)

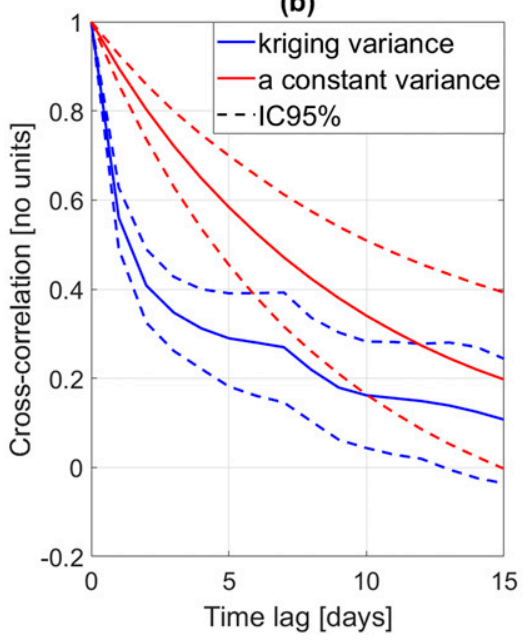

(c)

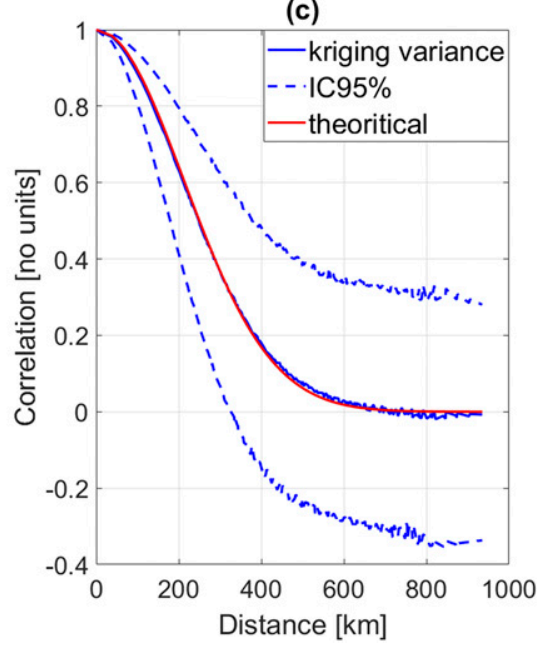

FIG. 3. Impact of using the kriging variance (which is not constant in time) on the temporal correlation of (a) precipitation and (b) temperature, as well as (c) the spatial correlation of perturbations.

using a constant variance (in red) with the correlations we obtain when using the kriging's variance (in blue). For temperature (Fig. 3a), the temporal dependency is lightly impacted, whereas for precipitation (Fig. 3b), the loss of temporal dependency is notable. This can be explained by the high temporal variability of precipitation, whereas temperature is highly temporally correlated. Unlike temporal dependency, spatial coherence appears to have no consequence on the resulting correlation for precipitation (Fig. 3c). Indeed, only the diagonal terms in the covariance matrix are modified during the construction of the Gaussian random field.

\section{4) AVOIDING FILTER DEGENERACY: RESAMPLING PROCEDURE}

A resampling procedure is used to avoid the degeneracy of the particle filter. The goal is to replace "bad" particles (with negligible weight because they correspond to simulated SWE values that are very far from observed values) by "good" particles (with high weights). This ensures that a good approximation of the a posteriori distribution is maintained across time. There are different ways to resample particles that are very well documented in the scientific literature. The most common procedure is the Sampling Importance Resampling (SIR) scheme introduced by Gordon et al. (1993) or the residual procedure (RES) introduced by Douc and Cappé (2005). Leisenring and Moradkhani (2011) proposed to use the weighted random resampling with replacement (WRR) in a SWE estimation context. All three procedures have been successively implemented and led to very similar estimated SWE (not shown here). In the remaining of the paper, only the SIR procedure is performed and the resampled particles are given equal weights. Although many authors, such as Doucet and Johansen (2009), resample only when the $N_{\text {eff }}$ falls below $N_{P} / 2$, here we chose a more restrictive threshold of $0.8 N_{P}$, like in Magnusson et al. (2017). Indeed, during preliminary testing of the filter setup, we observed a degradation of the performances (in terms or RMSE, for instance) when resampling was performed for $0.7 N_{P}$ or less. However, increasing the threshold to $0.9 N_{P}$ compared to $0.8 N_{P}$ did not improve performances any further.

\section{c. The spatial particle filter}

Meteorological input data are available over the studied area on a $0.10^{\circ}$ grid. Consequently, the snow model can provide a spatial distribution of SWE at the same resolution. With the perturbation procedure described in section $3 b(2)$, several particles can generate a possible value of SWE for each grid point (prediction step of the particle filter). Nevertheless, the updating step, which consists of estimating the particle weights via their likelihood requires an observation [Eq. (7)]. In our context, which is typical of large Nordic areas, observations are only available for a few points in space and those points do not necessarily correspond to grid points (they rarely do, in fact). Consequently, a crucial question to ask is, how can we perform the updating step of the particle filter at locations where no observations are available?

A first option would be to interpolate the observed SWE over the entire studied area using the same grid as meteorological inputs. This could be done using a classical, well-known spatial interpolation method, such as universal kriging (UK) or inverse distance weighting (IDW). UK is intuitively appealing, since it also evaluates 
the uncertainty of each interpolated value via the kriging variance. This uncertainty can account for measurement noise and all other sources of uncertainty. However, interpolators such as kriging have a smoothing effect problem. In addition, in the present study, UK poses additional challenge as the measurements are done asynchronously over all sites, with difference in timing for up to 4 days. Generally, the observed SWE is available for all sites at the end of each snow survey, and each survey typically extends over a week.

In this paper, we propose a simple solution to this problem of asynchronous observations. It consists in interpolating the weights of the particles, rather than observed SWE. This idea stems from the fact that because of the abovementioned spatial coherence considerations, we are already generating similar perturbations for neighboring points (see Fig. 2). We therefore make the following hypothesis: if a perturbation (applied both on input data and on state variables) leads to a good candidate value for SWE at site $s$, then a similar perturbation also allows us to obtain a good candidate value for SWE at points in the vicinity of site $s$. In other words, if a particle is good (bad) for a site, then it is also good (bad) for its neighborhood. To verify this hypothesis, we used the classical variogram introduced by Matheron (1963). For each particle (500), an empirical variogram was fitted based on the spatial distribution of particle weights, after the data assimilation step (update of the weights). This variogram is therefore based only on stations for which a new measurement occurred during the week. After an "updating week," we have 500 empirical variograms (one for each particle), and the filter is reinitialized (all particles are attributed an identical weight of $\left.1 / N_{P}\right)$. During the study period of 2000-16, there are 84 updating weeks (a week was considered as an updating week only if a new snow survey becomes available for at least $70 \%$ of all stations). The median of the $500 \times 84$ empirical variograms is illustrated in Fig. 4, which allows for judging the spatial dependence of the particle weights. The red curve indicates that weights are spatially independent if perturbations are not spatially correlated (as expected). The blue curve represents the situation where the perturbations applied to the meteorological inputs included a spatial correlation [Eq. (10)]. This blue curve shows that particle weights now indeed possess a spatial structure, which originates from the spatially correlated perturbations applied to the meteorological inputs to the snow model.

In practice, local particle weights are updated precisely at the date where observed SWE becomes available. The spatial interpolation of particle weights is then performed by IDW at the end of the week, in order to include the maximum number of observed SWE in the

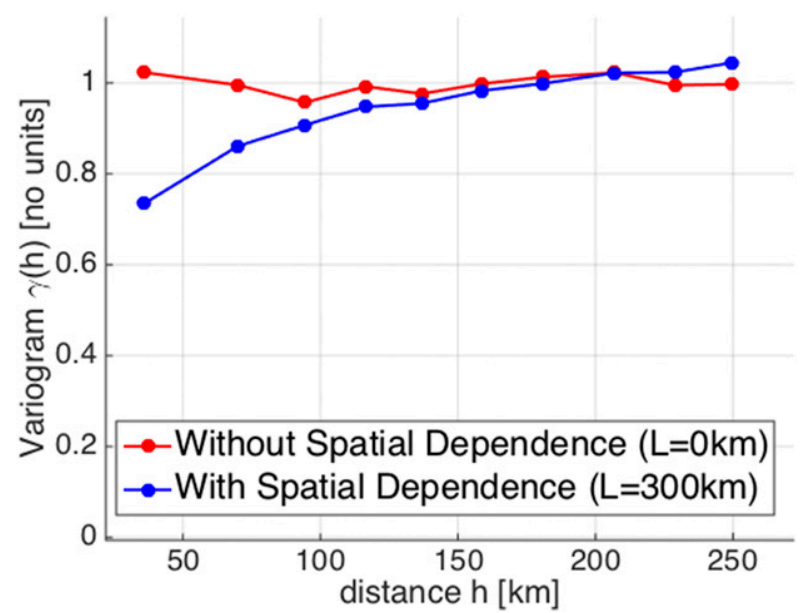

FIG. 4. Average empirical variogram of particle weights obtained using perturbations with (blue) and without (red) spatial dependency.

process. Finally, the resampling step is performed independently for all grid points.

\section{d. Optimal interpolation error as a benchmark}

For comparison purpose, results of the spatial particle filter will be compared to the operational snow assimilation scheme currently used at the DEH. The latter consist of direct insertion of the result of optimal interpolation of the SWE into the snow model.

$\mathrm{OI}$ is used to combine observational data with data obtained from a background field by exploiting the spatial correlation of the differences between observation (SWE provided by the snow survey measurements) and the background field (SWE provided by the snow model). The OI method calculates a correction to be applied to a background field from a linear combination of the differences between observations and the model at neighboring sites. For a given day, the estimation of a target site $z_{t}$ is given by

$$
z_{t}=x_{t}+\sum_{i=1}^{N} w_{i}\left(y_{i}-x_{i}\right) \text {, }
$$

where $N$ is the number of observation sites, $x_{t}$ is the value of the background field at the target site, $x_{i}$ is the value of the background field at the reference site $i$, and $y_{i}-x_{i}$ represents the difference between observation and the background field at site $i$. The weights $w_{i}$ are obtained by solving

$$
\left[\mathbf{R}+\mathbf{I} \frac{\sigma_{0}^{2}}{\sigma_{b}^{2}}\right] \mathbf{w}=\mathbf{r},
$$

where $\mathbf{R}$ (resp. $\mathbf{r}$ ) is the correlation matrix $N \times N$ (resp. $N \times 1$ ) of the error of the background field between the 
reference sites (resp. and the target site), $\mathbf{I}$ is the diagonal unit matrix $N \times N, \sigma_{0}^{2}$ is the variance of observation error, and $\sigma_{b}^{2}$ is the variance of the error of the background field.

Equation (15) stems from the nondimensional form of Garand and Grassotti (1995) and assumes a spatially uniform error for observations and background fields as well as uncorrelated errors for observations. Besides, it highlights the importance of the ratio between the variance of the observation error and the variance of the model error in the determination of weights. For this study, the DEH operational value of 0.15 was used.

The evaluation of $\mathbf{R}$ and $\mathbf{r}$ requires finding a function describing the correlation of the error of the background field as a function of the separation distance $h$. The following form was used to describe the correlation of error:

$$
\rho(h)=\left(1+\frac{h}{L_{\mathrm{OI}}}\right) \exp \left(-\frac{h}{L_{\mathrm{OI}}}\right),
$$

where the value of $L_{\mathrm{OI}}=22.6 \mathrm{~km}$ was obtained through a fit with experimental data.

Since OI exploits the spatial structure of the model error, it performs best when many observations are available at the assimilation time. To overcome the asynchronicity of snow survey across the territory, the snow model is used to artificially bring all measurements to the assimilation step which occurs weekly on Saturday at midnight. This is done by directly inserting snow measurement into the snow model and by simulating snowpack melt and snow accumulation up to the assimilation time step. After each assimilation step, the SWE estimates obtained from Eq. (15) are directly inserted into the snow model states.

\section{Results}

We first wanted to test the ability of the particle filter to estimate SWE at locations where observations are available (see section 4a). After all, this is really the most basic requirement in terms of performance and the application of the filter at unobserved locations is a step further. This amounts to assessing the temporal abilities of our particle filter since the spatial aspect is not considered in this first verification. Our benchmark here is direct insertion. It is a very severe benchmark, since direct insertion amounts to giving full credit to observed SWE and evaluating performance is also evaluated by comparing simulations with observations. Hence, it is expected that the particle filter will, at best, perform as good as direct insertion in the short term. However, one clear advantage of the particle filter (or any structured data assimilation method that alters state variables in a coherent manner) is that its effect should be more durable that direct insertion at longer lead times.

In section $4 \mathrm{~b}$, a cross validation is applied on three neighboring observation sites to illustrate the spatial behavior of the filter. This cross validation is performed in turn for scenarios where the predicted SWE is underestimated, and then overestimated, compared to the observation.

To also evaluate the ability of the filter to estimate SWE at unobserved location (spatial abilities), we resort to leave-one-out (LOO) cross validation and we also validate using the independent dataset.

In section $4 c$, the LOO cross validation is applied to all sites in turn. To some extent, this allows us to judge the ability of the filter to estimate SWE at unobserved locations. Indeed, the LOO cross validation specifically aims at data sparse situations, as it is the case here (e.g., Arlot and Celisse 2010; Stone 1974). It is a form of $k$-fold cross validation, with $k$ equal to one. We then perform a final verification using the independent dataset.

Performance is compared to the current operational method (see section 3d). Finally, we illustrate the spatial distribution of SWE as obtained with the spatial particle filter and optimal interpolation in section $4 \mathrm{~d}$.

\section{a. At-site SWE estimation (temporal performance)}

Here, we consider four different methods to estimate at-site SWE. The first one, named Open Loop, consists in running the snow model without taking into account SWE observations at all. The second one, named direct insertion (DirIns), consists in replacing the predictions of the snow model with observed SWE as they become available. The third one, denoted PF, is our implementation of the particle filter as described in section 3b. Finally, we also include an alternative implementation of the particle filter, which we refer to as PFnoTempoCor. In this implementation, no temporal dependency is included [ $\alpha=0$ in Eq. (11)]. Hence, this will allow us to assess the importance of considering temporal dependency in the filter.

The different methods are applied at the 88 snow survey sites mentioned in section $2 \mathrm{~b}$ and shown in blue in Fig. 1, in the period 2000-16. This corresponds to the solid lines on Fig. 5. The dashed lines correspond to the results for the additional sites shown in red in Fig. 1. For each method, the accuracy is assessed using the RMSE and NSE metrics. Those metrics are calculated for each site just before the updating step. After the updating step, the SWE predicted by direct insertion are equal to the observed SWE values. The metrics show the ability to predict SWE after 2 weeks without data assimilation. The metrics are calculated with approximately 60 SWE measurements for each site. For the two variants of the 
(a)

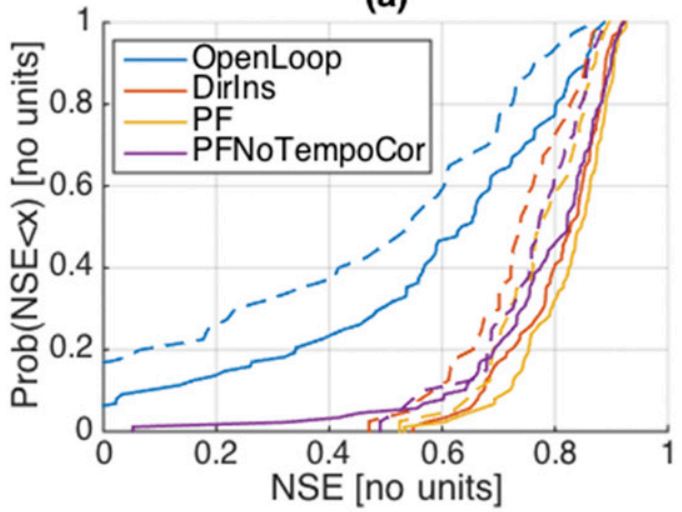

(c)

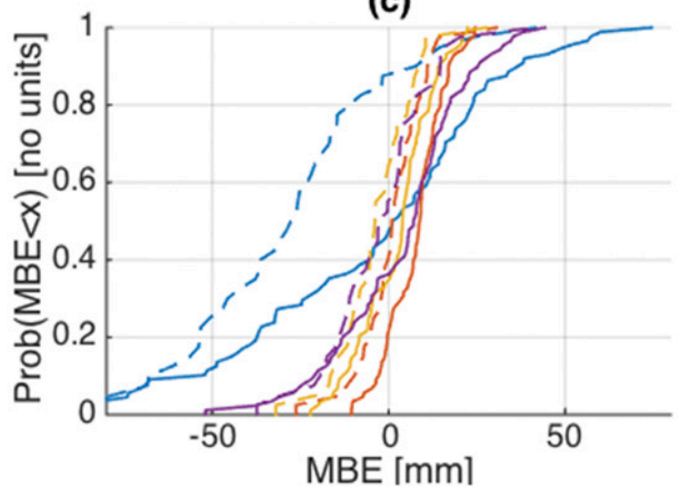

(b)

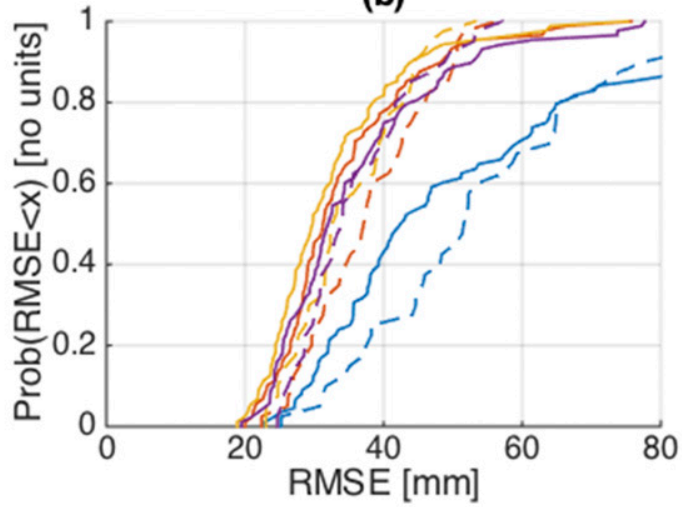

(d)

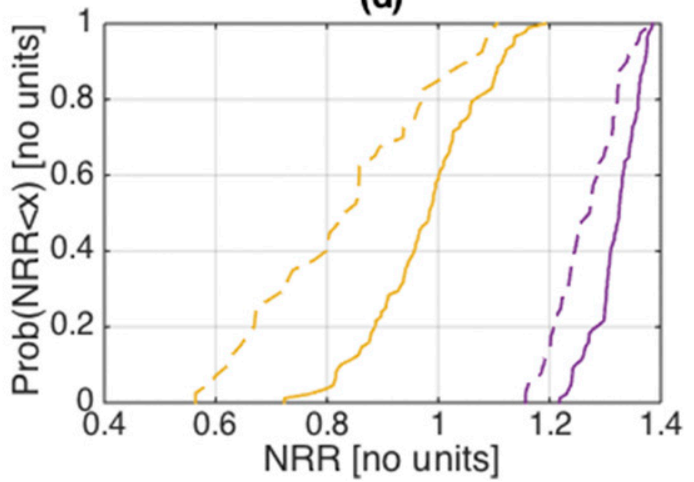

FIG. 5. Local performance metrics for Open Loop (blue), DirIns (red), and PF with (yellow) and without (purple) temporal correlation during the period 2000-16 for the calibration sites (solid lines) and for the validation sites (independent dataset; dashed lines). The RMSE and the MBE are in millimeters.

particle filter described above, the NRR is also calculated to measure the dispersion of the ensemble, for each of the 88 sites.

Figure 5 illustrates the empirical cumulative distribution of the local performance metrics. As expected, performing data assimilation greatly improves SWE predictions. Indeed, for assimilation methods, the NSE exceeds 0.8 for the majority of sites, against 0.6 for the Open Loop simulation. Moreover, data assimilation is able to correct predicted SWE at specific points in space where the snow model is not fully adapted, perhaps because of the global calibration of parameters (i.e., sites with NSE $<0$ ). In addition, the temporal dependency in perturbations does improve the accuracy of the filter, even if the temporal dependency is impacted by the kriging variance (see Fig. 3), and becomes essential at locations where the snow model performs poorly in Open Loop. In addition, it allows us to obtain a good dispersion of the ensemble (Fig. 5d). According to Thiboult and Anctil (2015), a good dispersion is characterized by a NRR around $1( \pm 0.2)$. The accuracy of DirIns and PF is high, since $90 \%$ of sites have a NSE superior to 0.7 after 2 weeks without observation (and therefore no data assimilation). The median of PF's particles appear globally more accurate than DirIns. The corresponding bias (defined by MBE in Table 2) is normally distributed and centered in 0 , whereas DirIns slightly overestimates SWE. Finally, by comparing the solid and dashed lines, one can notice that performances decrease for the additional dataset. This is not surprising, as the blue and red sites displayed in Fig. 1 are not evenly distributed in space. The snow model was calibrated using the blue sites, and it is not unusual for a model to reach a lesser (but still satisfying) performance for data it was not calibrated on.

\section{b. The spatial particle filter applied on three neighboring sites}

In this section, we focus on the spatial particle filter (denoted SPF) and its ability to transfer information from sites where there are observations to sites where no observations are available. We consider three sites, all located in an area called the Montmorency forest $\left(47.29^{\circ} \mathrm{N}, 71.16^{\circ} \mathrm{W}\right)$. Snow surveys are performed regularly at all three sites. The distance between those sites 
does not exceed $1 \mathrm{~km}$ and their elevation lies between 775 and $850 \mathrm{~m}$ MSL. Due to the limited spatial resolution of gridded meteorological observations, the three sites are forced by the same input data. Temperature slightly differs, since a thermal gradient of $0.5^{\circ} \mathrm{C}$ is applied.

The SPF was tested on these three sites with a leaveone-out cross validation to estimate SWE at a target site, only observed SWE at the two neighboring sites is considered. Note that snow surveys at these three sites always occurs on the same day. Thus, observed SWE can be interpolated at the target site (instead of interpolating the weights) and PF can be applied using the interpolated SWE. This method is the inverse distance weighted particle filter (IDWPF). IDW is used here because there are only three points, which is clearly not sufficient to perform kriging.

The Open Loop simulation and the SWE series produced using the PF have been also produced for the 2000-16 period and the corresponding performance metrics are summarized in Table 3. At those locations, the snow model tends to underestimate $\mathrm{SWE}(\mathrm{MBE}<0$ for Open Loop). Despite the fact that the same input data is used and the high variability of SWE between sites, the PF provides an accurate estimation of SWE at each site and a good ensemble dispersion. Indeed, there are significant differences between at-site observed SWE, which can exceed $30 \%$ in spite of the proximity of those three sites (less than $1 \mathrm{~km}$ ). This fact underlines the high spatial variability of SWE and the inherent limitation is the common working hypothesis, which consists of assuming that a point measurement of SWE is representative of the real SWE over an entire grid cell. This is even truer with a fairly coarse $0.1^{\circ}$ resolution. The use of SPF allows us to improve SWE estimations noticeably for two sites out of three. At site number three, where the SPF is less accurate than the Open Loop simulation, the loss of performance is limited compared to the gain at the two other sites. The SPF and the IDWPF show similar performances and it can be seen that, for neighboring sites, particle weights carry almost as much information as observations themselves. This is an encouraging remark, which leads us to think that a carefully designed spatial particle filter could be useful to infer SWE at unobserved locations. This will be tested in section $4 \mathrm{c}$.

Figure 6 illustrates the SWE estimated by the three methods during the winter of 2012-2013 at each site. Figure $6 \mathrm{~d}$ zooms on the January-February period to show an example of the trajectory of the particles after a resampling step. Interpolating particle weights enables us to transfer the available information to target sites. Indeed, the SWE values estimated by the SPF (in green) differ from those simulated in Open Loop (in blue). Since
TABLE 3. Local performance metrics for SWE estimations according to: Open Loop, PF, IDWPF, and SPF for the 2000-16 period at sites located in the Montmorency forest. The performance assessment is always performed after the updating step. NSE and NRR are unitless.

\begin{tabular}{|c|c|c|c|c|}
\hline Elevation (m) & & 858 & 825 & 777 \\
\hline Methods & Scores & Site 1 & Site 2 & Site 3 \\
\hline \multirow[t]{3}{*}{ Open Loop } & NSE & 0.63 & 0.28 & 0.53 \\
\hline & RMSE (mm) & 91 & 122 & 93 \\
\hline & $\operatorname{MBE}(\mathrm{mm})$ & -48 & -100 & -18 \\
\hline \multirow[t]{4}{*}{$\mathrm{PF}$} & NSE & 0.97 & 0.96 & 0.82 \\
\hline & RMSE (mm) & 28 & 26 & 55 \\
\hline & $\operatorname{MBE}(\mathrm{mm})$ & -1 & -10 & -8 \\
\hline & NRR & 1.04 & 1.01 & 1.22 \\
\hline \multirow[t]{4}{*}{ SPF } & NSE & 0.79 & 0.60 & 0.44 \\
\hline & RMSE (mm) & 65 & 89 & 98 \\
\hline & $\operatorname{MBE}(\mathrm{mm})$ & 8 & -79 & 54 \\
\hline & NRR & 1.12 & 1.22 & 1.25 \\
\hline \multirow[t]{4}{*}{ IDWPF } & NSE & 0.80 & 0.61 & 0.37 \\
\hline & RMSE (mm) & 62 & 89 & 103 \\
\hline & $\operatorname{MBE}(\mathrm{mm})$ & -4 & -73 & 60 \\
\hline & NRR & 1.21 & 1.27 & 1.26 \\
\hline
\end{tabular}

the sites are equidistant, particle weights at the target site are equally influenced by the observed SWE at the two neighboring sites. During the resampling step, particles that are too far from the corresponding observation are removed and replaced it by good particles. After this resampling step, the concentration of particles between the two available observed SWE thus becomes higher. That explains why the SPF performs especially well at site number 1 , since this site lies exactly between the two neighboring sites. Inversely, for site number 3 , which receives less precipitation on average than the two other ones, the SPF actually degrades SWE estimation from the snow model, because assimilation is based on two points that receive more snow. Furthermore, the snow model tends to underestimate SWE at this location. The $90 \%$ confidence interval of the SPF is higher than the one for $\mathrm{PF}$, since it is deduced from two observed SWE locations instead of only one. However, according to the NRR score, the ensemble generated by the SPF does not have enough spread, since the score exceeds 1 at each site.

\section{c. Spatial performance: Leave-one-out cross validation and validation on a new dataset}

The SPF was tested on the first 88 sites of the study area (blue sites, Fig. 1) using a leave-one-out cross validation. The Open Loop simulation as well as the values obtained by direct insertion with optimal interpolation method DirIns-OI (see section 3d) serve as benchmarks for comparing and analyzing the results.

Figure 7 illustrates the empirical cumulative distribution of the performance metrics computed for 
(a)

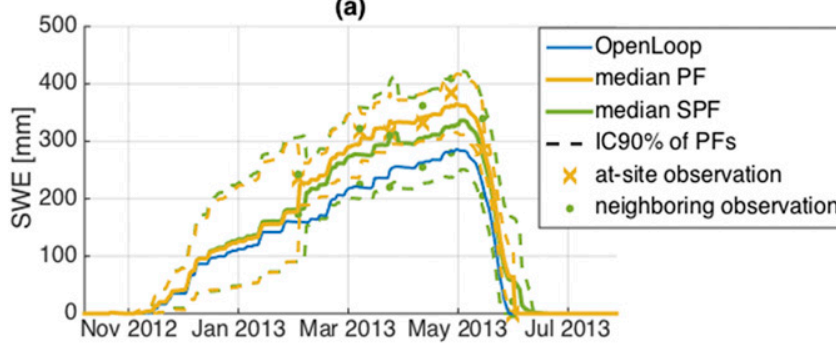

(c)

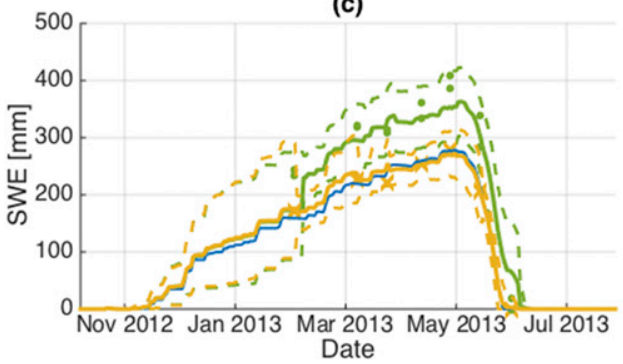

(b)

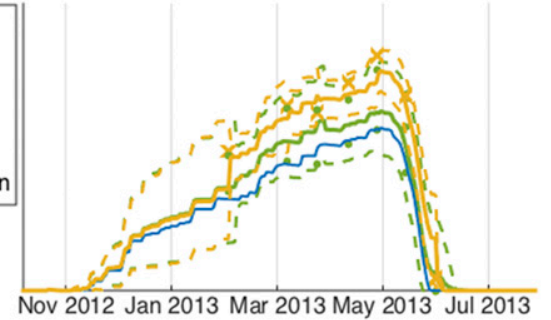

(d)

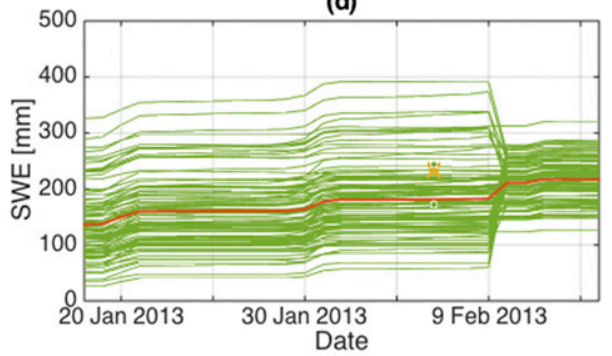

FIG. 6. (a)-(c) SWE as simulated in Open Loop (blue), with the PF (yellow), and with the SPF (green) during the winter of 2012/13. (d) The trajectories of 100 particles (among 500) after the first observation of the winter (at site number one).

each measurement site and for the three methods. Note that those metrics assess the ability of the data assimilation method to estimate SWE after about 2 weeks without any observations (like in section 4a) since the updating step occurs at the end of the snow survey week.

Globally, both the SPF and DirIns-OI are successful at decreasing the RMSE. This decreases the amount (a)

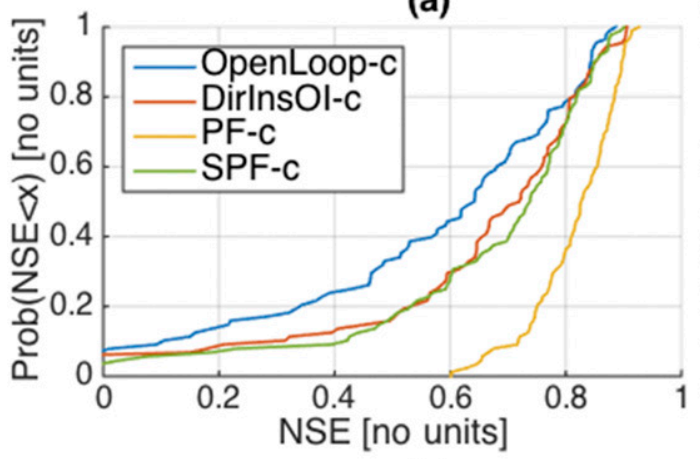

(c)

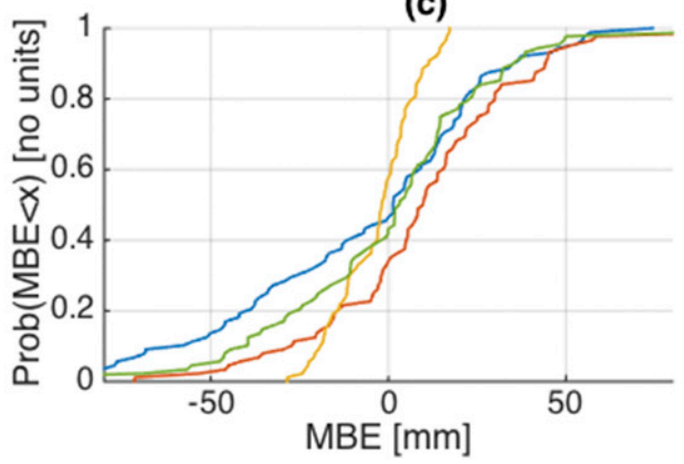

(b)

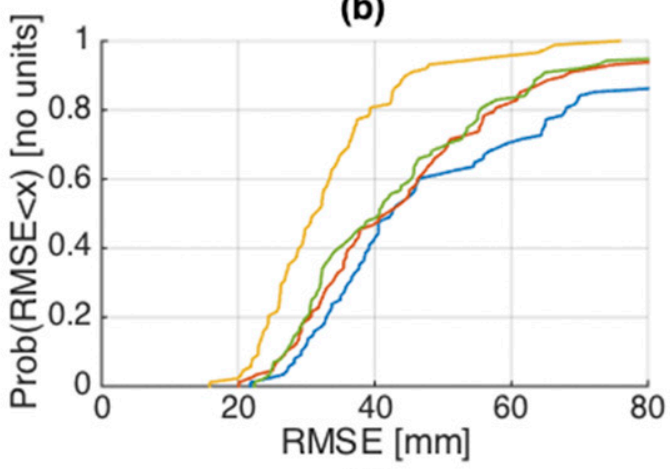

(d)

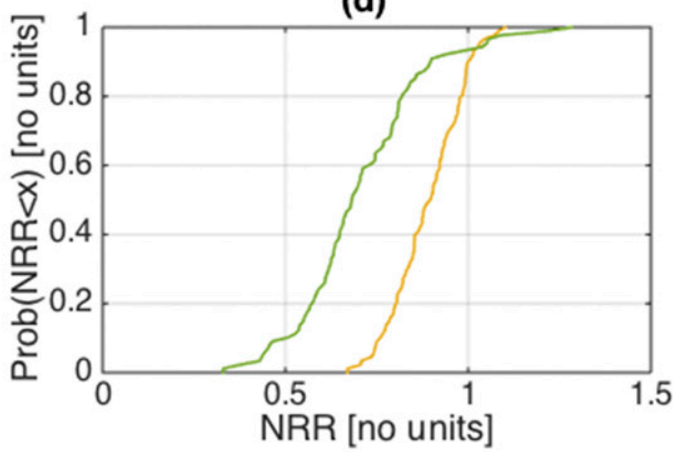

FIG. 7. Local performance metrics in a leave-one-out cross-validation configuration (calibration dataset) for the Open Loop simulation (blue), direct insertion with DirIns-OI (red), and the SPF (green) during the period of 2000-16. Local performance metrics for the PF (yellow) are also illustrated for comparison. The RMSE and the $\mathrm{MBE}$ are given in millimeters. 
(a)

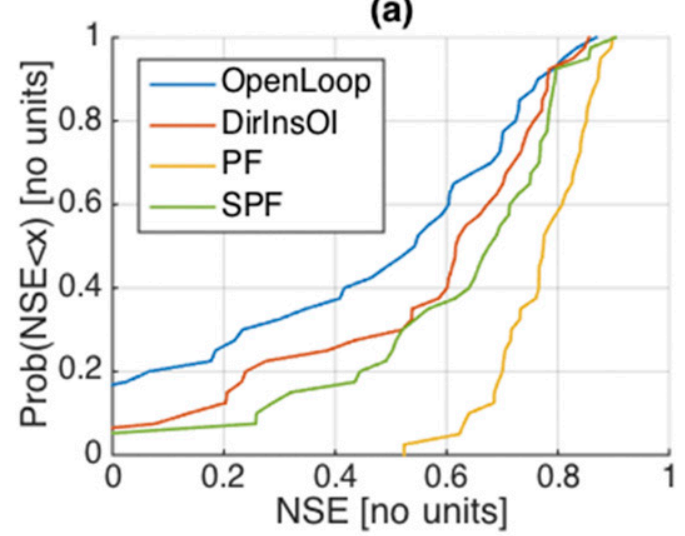

(c)

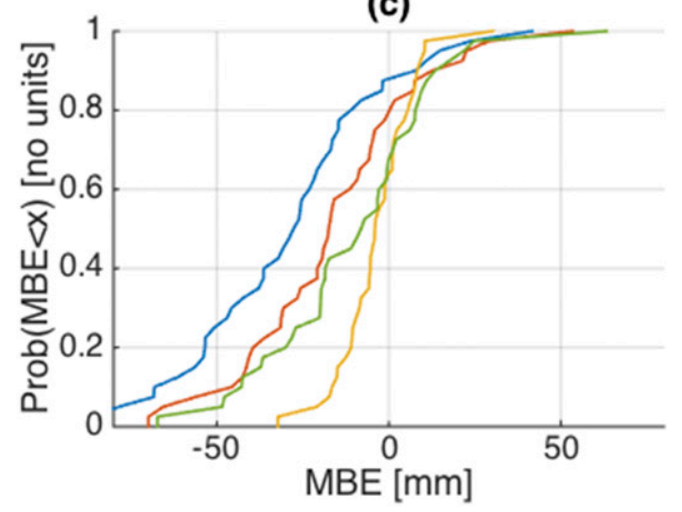

(b)

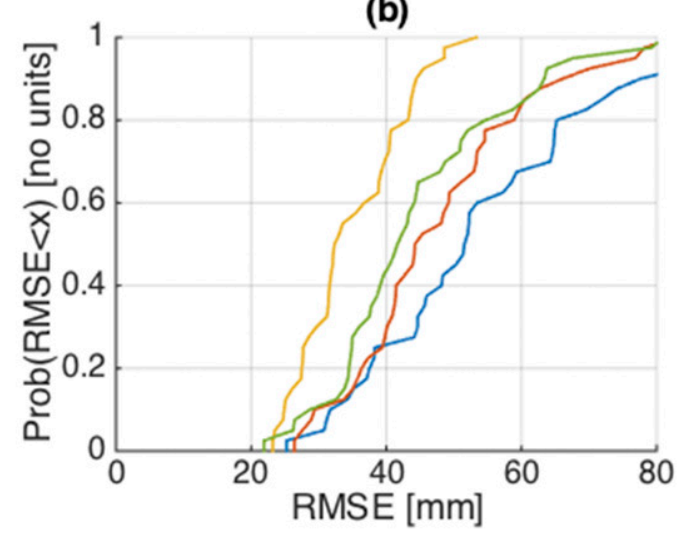

(d)

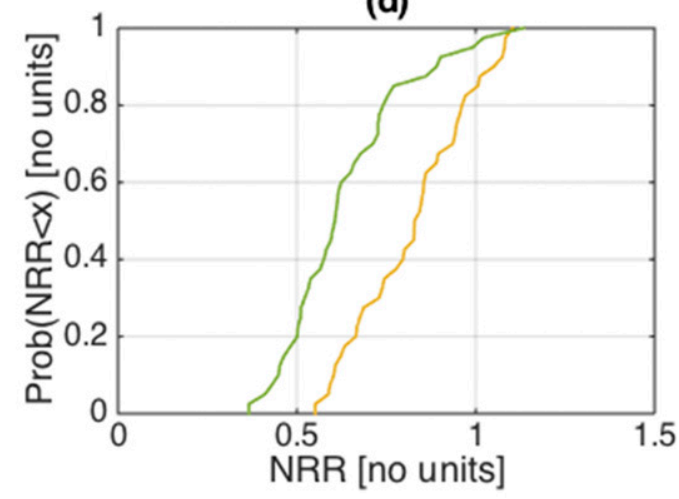

FIG. 8. Local performance metrics for the independent dataset, for the Open Loop simulation (blue), direct insertion with DirIns-OI (red) and the SPF (green) during the period of 2000-16. Local performance metrics for the PF (yellow) are also illustrated. The RMSE and the MBE are given in millimeters.

to approximately $8 \mathrm{~mm}$ in the study area $(\sim 50 \mathrm{~mm}$ for DirIns-OI and SPF vs $\sim 58 \mathrm{~mm}$ in Open Loop) and increases the NSEg (0.77 for DirIns-OI and SPF vs 0.69 in Open Loop).

Locally, the RMSE is reduced for more than $90 \%$ of sites when data assimilation is applied, and this decrease exceeds $30 \mathrm{~mm}$ for $25 \%$ of sites. This gain seems not to be linked with the number of sites in the neighborhood of the target site or with the distance with the closest site. As expected, the loss of performance is quite noticeable compared with the PF, for which at-site observations are available. In addition, the SPF seems slightly more accurate than DirIns-OI. For $60 \%$ of sites, the difference between the local RMSE of the two methods does not exceed $5 \mathrm{~mm}$. Contrary to DirIns-OI, the bias of the SWE series after data assimilation using the SPF is centered on 0 . Finally, the ensemble formed of all the particles used with the SPF has more spread than the ensembles obtained with the PF, but it remains acceptable for the majority of sites (NRR $>0.7$ ).

Using interpolation methods to transfer available information into target site improves greatly the SWE estimation on the study area.
Figure 8 shows the same performance metrics but at initially unobserved locations. Indeed, these are the observation sites shown in red in Fig. 1, for which data were provided later on during the study. Therefore, they can be considered as initially "unobserved" sites, since they were never used for the calibration of the snow model or for the initial assessment of the performance of the particle filter. As can be seen on this figure, all performance metrics indicate a superior performances of the SPF (in green) compared not only to the Open Loop simulation, but also to DirIns-OI (i.e., higher NSE values, lower RMSE and MBE closer to 0 ). This indicates that the proposed PF method can indeed provide a good (although certainly not perfect) estimation of SWE at unobserved locations.

\section{d. Spatial distribution of $S W E$}

Both the SPF and DirIns-OI have been applied over the entire study area at a $0.1^{\circ}$ resolution. Therefore, all available sites were taken into account. Figure 9 illustrates the spatial distribution of SWE as simulated by these methods before and after the first updating step 


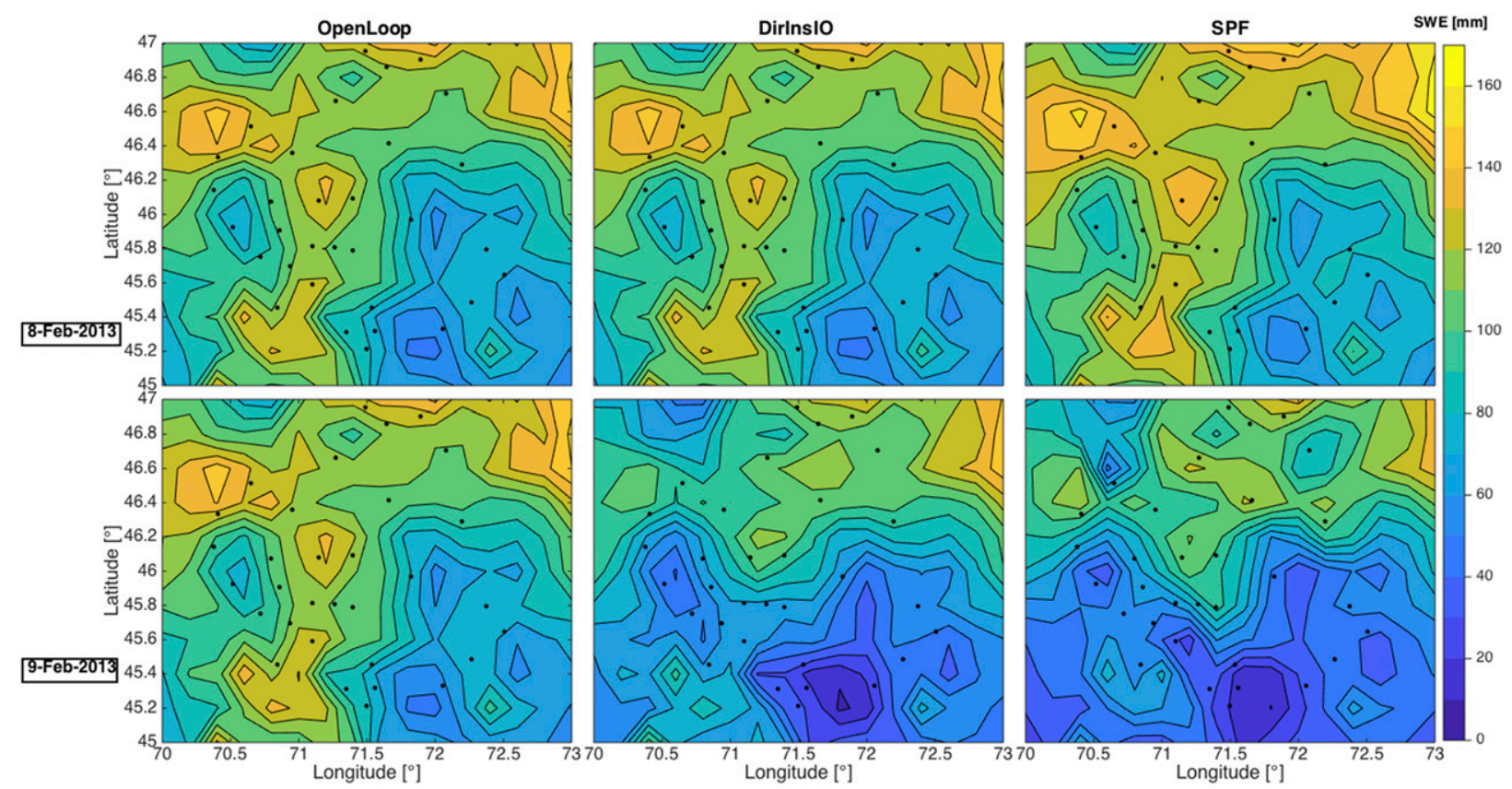

FIG. 9. The spatial distribution of (left) SWE, (center) DirIns-OI, and (right) the median of the SPF's particles (top) before and (bottom) after the first updating step of winter 2012/13 (9 Feb 2013). Points of observation for SWE are identified with black dots.

of the winter 2012/13. For visibility, we restricted Fig. 9 to a smaller representative portion of the study area.

Before the updating step, the spatial distribution of SWE is equivalent to the one obtained with the Open Loop simulation and with DirIns-OI since at this date, no data assimilation has been performed yet since it is still only the beginning of winter (top row in Fig. 9). The median of the SPF particles corresponds to a SWE that is slightly superior to the Open Loop simulation (between 40 and $14 \mathrm{~mm}$, depending on grid points). The bias can be explained by the process noise. More specifically, it originates from the perturbations applied on precipitation. Indeed, with the Gaussian additive noise, random perturbations add precipitation more frequently than removing it since, as opposed to temperature, precipitation cannot be negative. Obviously, when it does not rain or snow, one cannot remove precipitation. However, if rain or snow is falling, we can both add or remove precipitation.

For both data assimilation methods (SPF and DirInsOI), the updating step modifies the spatial distribution of SWE noticeably (between -30 and $80 \mathrm{~mm}$ ) for, a priori, a better accuracy. DirIns-OI is directly linked with the observation, since it interpolates the error between the model and observation, whereas, for the SPF, data assimilation is performed through model perturbations and during the interpolation of particle weights. There is thus no interpolation of the variable of interest (SWE) as such. Consequently, given their nature, the two methods propose two different series of SWE estimations. The differences are centered on $3 \mathrm{~mm}$ and range from -30 to $30 \mathrm{~mm}$. Still, there are also many similarities between the two methods, and the main patterns in SWE distributions are shared by the two methods. This similarity confirms that interpolating particle weights, instead of interpolating SWE, allows us to preserve the spatial coherency of information throughout the data assimilation process with the SPF.

\section{Summary and discussion}

In this paper, a particle filter was implemented to estimate the spatial distribution of the snow water equivalent (SWE) in a $600000 \mathrm{~km}^{2}$ portion of the province of Quebec, Canada.

First, a snow model forced by meteorological observations was calibrated with SCE-UA (section 2e). Then, a particle filter was implemented (see section 3). In this implementation, input perturbations are correlated both in space and time. Their magnitudes is imposed by the kriging's variance. First, the performance of this particle filter was assessed locally (meaning for each measurement site separately), leading to good performances in the estimation of at-site SWE. In fact, the performance was very similar to a direct insertion method (see section 4a), with the added benefit of reducing the bias. The main contribution of this paper, however, is in proposing to interpolate the particle weights, 
in order to be able to apply the filter at locations where no observations are available (section 3c). A leave-one-out cross validation showed that using this spatial particle filter to estimate SWE outperformed control simulations over the study area, and validation of the proposed method for an independent dataset has showed that it can outperform actual operational method based on direct insertion and optimal interpolation (section 4c). Despite of their conceptual differences, the interpolation of particle weights or the interpolation of model errors lead to very similar SWE values and spatial patterns. However, an advantage of the SPF is that no interpolation of the variable of interest is needed to estimate its value. Indeed, the information is rather transferred from one grid point to another through the particle weights. This particularity can be very useful in cases where the interpolation of the variable of interest is critical, such as when simulating river discharge.

The spatial particle filter is not difficult to implement and could easily be transferred to other studies area, provided that adequate data is available (see below for limitations). In this paper, inverse distance weighting was used to interpolate the particle weights, but other interpolation methods could also be applied. Kriging, for instance, could be an alternative, but then one would have to evaluate different variogram options. Some possibilities include fitting a theoretical variogram repeatedly at each updating step, or to fit a unique variogram that would remain fixed throughout time, for example as in Fig. 4. However, during this study, preliminary tests showed that the choice of a particular interpolation method has very limited impact on data assimilation performance. Note that, before applying the SPF, model perturbations must be spatially correlated, and the spatial correlation of particle weights must also be verified.

Generally, the hyperparameters that control the magnitude of the process noise are fine-tuned. In this study, only the hyperparameter that controls the model state perturbation was tuned since the perturbations on meteorological observations (snow model's input data) were imposed by the kriging variance. This choice was motivated by a desire to consider perturbations that are as objective and close to the "real" uncertainty of meteorological observations. While we recognize that this could still be improved and that kriging variance does not necessarily encompass all possible uncertainties of observed precipitation and temperature, in our opinion this is still more objective than artificially "fitting" the uncertainty of input data until it meets a certain level of performance for the particle filter and conclude that this must therefore be the true uncertainty. A drawback however is that the kriging variance vary in time and space, which leads to simulated perturbations that are slightly less temporally correlated as expected. This is especially true for precipitation (see Fig. 3). A particle filter was tested in which the process noise was constant in space and time. The best configuration was a multiplicative uniform noise between 0.7 and 1.3 for precipitation and an additive uniform noise between $-3^{\circ}$ and $+3^{\circ} \mathrm{C}$ for temperature, which correspond to lower uncertainties than those imposed by the kriging variance in most locations (especially for precipitation). This configuration led to very similar performances than those of the PF used in this study in terms of accuracy and bias. Nevertheless, the ensemble dispersion was improved, since the distribution of the NRR varied between 0.95 and 1.2 (against 0.75 and 1.2 in this study). We can therefore suppose that the large ensemble dispersion is at least partly due to the perturbations applied on meteorological observations. A data driven approach, as proposed in Pathiraja et al. (2018), could be an interesting avenue to further characterize model uncertainty in the context of data assimilation. Indeed, a better characterization of model uncertainty could lead to substantial improvements of the PF's behavior.

Regarding computation time, the particle filter is much more time consuming than direct insertion. However, in addition to a better accuracy, the particle filter proposes an ensemble of SWE values for each grid point (rather than a single value), which allows us to compute an uncertainty interval around the value that is the most probable. The distribution of SWE in space is highly variable, even in small subgrids of the study area (see Fig. 6), so the particle filter makes uncertainty assessment possible.

In this paper, the same set of model parameters is used to simulate the snowpack at all grid points. Perhaps the most obvious next step for further improvement would be the application of the SPF in a dual state-parameter framework. In such a framework, both state variables and model parameters are updated, like in Moradkhani et al. (2005a). In the same way, perturbations applied on parameters should be spatially correlated, and the particle weights could be interpolated in order to propose a set of updated parameters even at locations where no observations are available.

During the resampling step, particles can be removed and resampled. Consequently, the spatial structure of particle weights can be lost. Indeed the trajectory of the particle $i_{P}$ at site $s_{i}$ may not correspond to the trajectory of the particle $i_{P}$ at site $s_{j}$, even if $s_{i}$ is close to $s_{j}$. Thus, in this case, interpolating particle weights brings no information, or in the worst case, introduces false information in the filter. In this study, this is not preoccupying since there are only about five SWE observations per year and thus the same number of updating steps. On average, 
the resampling step is performed three times per year. Moreover, the filter can be reset after each winter, as it is not in use during the summer anyway. Our tests have shown that, at the end of the winter, approximately $20 \%$ of the particles are spatially uncorrelated and that the sampling importance resampling (SIR) procedure appears as the best method to limit the loss of spatial coherence among the two others tested resampling procedures (WRR, RES). This percentage can drastically increase with the number of resampling occurrences. Thus, should someone use our method in a context where daily observations are available and data assimilation is also performed daily, the filter must be reset more frequently. In that regard, further studies could also involve proposing new resampling methods that include a spatial structure to remove or clone particles in a certain neighborhood.

Acknowledgments. The authors would like to express their gratitude to Hydro-Québec, SOPFEU, Rio-Tinto and the other partners of the Réseau Météorologique Coopératif québecois (RMCQ) for graciously providing an independent data set of SWE measurements on which to validate the proposed method.

\section{REFERENCES}

Abbaszadeh, P., H. Moradkhani, and H. Yan, 2018: Enhancing hydrologic data assimilation by evolutionary particle filter and Markov chain Monte Carlo. Adv. Water Resour., 111, 192-204, https://doi.org/10.1016/j.advwatres.2017.11.011.

Anderson, E. A., 2006: Snow accumulation and ablation modelSNOW-17. User's manual, NWS, 61 pp., http://www.nws.noaa.gov/ oh/hrl/nwsrfs/users_manual/part2/_pdf/22snow17.pdf.

Arlot, A., and A. Celisse, 2010: A survey of cross-validation procedures for model selection. Stat. Surv., 4, 40-79, https://doi.org/ 10.1214/09-SS054.

Barnett, T. P., J. C. Adam, and D. P. Lettenmaier, 2005: Potential impacts of a warming climate on water availability in snowdominated regions. Nature, 438, 303-309, https://doi.org/10.1038/ nature 04141.

Bergeron, O., 2017: Grilles climatiques quotidiennes du Réseau de surveillance du climat du Québec, Version 2 - Guide d'utilisation. Québec, ministère du Développement durable, de l'Environnement et de la Lutte contre les changements climatiques, Direction du suivi de l'état de l'environnement, $27 \mathrm{pp}$.

Burgess, T. M., and R. Webster, 1980: Optimal interpolation and isarithmic mapping of soil properties. Eur. J. Soil Sci., 31, 315331, https://doi.org/10.1111/j.1365-2389.1980.tb02084.x.

Clark, M. P., D. E. Rupp, R. A. Woods, X. Zheng, R. P. Ibbitt, A. G. Slater, J. Schmidt, and M. J. Uddstrom, 2008: Hydrological data assimilation with the ensemble Kalman filter: Use of streamflow observations to update states in a distributed hydrological model. Adv. Water Resour., 31, 1309-1324, https://doi.org/ 10.1016/j.advwatres.2008.06.005.

Dechant, C., and H. Moradkhani, 2011: Radiance data assimilation for operational snow and streamflow forecasting. Adv. Water Resour., 34, 351-364, https://doi.org/10.1016/j.advwatres.2010.12.009.
Dong, J., J. P. Walker, and P. R. Houser, 2005: Factors affecting remotely sensed snow water equivalent uncertainty. $R e$ mote Sens. Environ., 97, 68-82, https://doi.org/10.1016/ j.rse.2005.04.010.

Douc, R., and O. Cappé, 2005: Comparison of resampling schemes for particle filtering. Proc. Fourth Int. Symp. on Image and Signal Processing and Analysis, Zagreb, Croatia, IEEE, 64-69, https://doi.org/10.1109/ISPA.2005.195385.

Doucet, A., and A. M. Johansen, 2009: A tutorial on particle filtering and smoothing: Fifteen years later. The Oxford Handbook of Nonlinear Filtering, D. Crisan and B. Rozovskiı̆, Oxford University Press, 656-704.

Duan, Q., S. Sorooshian, and V. Gupta, 1992: Effective and efficient global optimization for conceptual rainfall-runoff models. Water Resour. Res., 28, 1015-1031, https://doi.org/10.1029/ 91WR02985.

Essery, R., S. Morin, Y. Lejeune, and C. B. Ménard, 2013: A comparison of 1701 snow models using observations from an alpine site. Adv. Water Resour., 55, 131-148, https://doi.org/ 10.1016/j.advwatres.2012.07.013.

Evensen, G., 1994: Sequential data assimilation with a nonlinear quasi-geostrophic model using Monte Carlo methods to forecast error statistics. J. Geophys. Res., 99, 10143-10162, https://doi.org/10.1029/94JC00572.

, 2003: The ensemble Kalman filter: Theoretical formulation and practical implementation. Ocean Dyn., 53, 343-367, https:// doi.org/10.1007/s10236-003-0036-9.

Fletcher, S. J., G. E. Liston, C. A. Hiemstra, and S. D. Miller, 2012: Assimilating MODIS and AMSR-E snow observations in a snow evolution model. J. Hydrometeor., 13, 1475-1492, https:// doi.org/10.1175/JHM-D-11-082.1.

Fortin, J.-P., R. Turcotte, S. Massicotte, R. Moussa, J. Fitzback, and J.-P. Villeneuve, 2001: Distributed watershed model compatible with remote sensing and GIS data. I: Description of model. J. Hydrol. Eng., 6, 91-99, https://doi.org/10.1061/ (ASCE)1084-0699(2001)6:2(91).

Garand, L., and C. Grassotti, 1995: Toward an objective analysis of rainfall rate combining observations and short-term forecast model estimates. J. Appl. Meteor., 34, 1962-1977, https://doi.org/10.1175/1520-0450(1995)034<1962:TAOAOR> 2.0.CO;2.

Goita, K., A. Walker, and B. Goodison, 2003: Algorithm development and use for the estimation of snow water equivalent in the boreal forest using passive microwave data. Int. J. Remote Sens., 24, 1097-1102, https://doi.org/10.1080/ 0143116021000044805.

Gordon, N. J., D. J. Salmond, and A. F. Smith, 1993: Novel approach to nonlinear/non-Gaussian Bayesian state estimation. IEE Proc., F, Radar Signal Process., 140, 107-113, https:// doi.org/10.1049/ip-f-2.1993.0015.

Leisenring, M., and H. Moradkhani, 2011: Snow water equivalent prediction using Bayesian data assimilation methods. Stochastic Environ. Res. Risk Assess., 25, 253-270, https://doi.org/10.1007/ s00477-010-0445-5.

Li, L., and S. Simonovic, 2002: System dynamics model for predicting floods from snowmelt in North American prairie watersheds. Hydrol. Processes, 16, 2645-2666, https://doi.org/ 10.1002/hyp.1064.

Liu, Y., C. D. Peters-Lidard, S. Kumar, J. L. Foster, M. Shaw, Y. Tian, and G. M. Fall, 2013: Assimilating satellite-based snow depth and snow cover products for improving snow predictions in Alaska. Adv. Water Resour., 54, 208-227, https://doi.org/10.1016/ j.advwatres.2013.02.005. 
López-Moreno, J., and D. Nogués-Bravo, 2006: Interpolating local snow depth data: An evaluation of methods. Hydrol. Processes, 20, 2217-2232, https://doi.org/10.1002/hyp.6199.

, S. R. Fassnacht, S. Beguería, and J. B. P. Latron, 2011: Variability of snow depth at the plot scale: Implications for mean depth estimation and sampling strategies. Cryosphere, 5, 617629, https://doi.org/10.5194/tc-5-617-2011.

Magnusson, J., D. Gustafsson, F. Hüsler, and T. Jonas, 2014: Assimilation of point SWE data into a distributed snow cover model comparing two contrasting methods. Water Resour. Res., 50, 7816-7835, https://doi.org/10.1002/2014WR015302.

— A. Winstral, A. S. Stordal, R. Essery, and T. Jonas, 2017: Improving physically based snow simulations by assimilating snow depths using the particle filter. Water Resour. Res., 53, 1125-1143, https://doi.org/10.1002/2016WR019092.

Marks, D., and A. Winstral, 2001: Comparison of snow deposition, the snow cover energy balance, and snowmelt at two sites in a semiarid mountain basin. J. Hydrometeor., 2, 213-227, https:// doi.org/10.1175/1525-7541(2001)002<0213:COSDTS >2.0.CO;2.

Matheron, G., 1963: Principles of geostatistics. Econ. Geol., 58, 1246-1266, https://doi.org/10.2113/gsecongeo.58.8.1246.

Moradkhani, H., K.-L. Hsu, H. Gupta, and S. Sorooshian, 2005a: Uncertainty assessment of hydrologic model states and parameters: Sequential data assimilation using the particle filter. Water Resour. Res., 41, W05012, https://doi.org/10.1029/ 2004WR003604.

, S. Sorooshian, H. V. Gupta, and P. R. Houser, 2005b: Dual state-parameter estimation of hydrological models using ensemble Kalman filter. Adv. Water Resour., 28, 135-147, https:// doi.org/10.1016/j.advwatres.2004.09.002.

— C. M. DeChant, and S. Sorooshian, 2012: Evolution of ensemble data assimilation for uncertainty quantification using the particle filter-Markov chain Monte Carlo method. Water Resour. Res., 48, W12520, https://doi.org/10.1029/2012WR012144.

Nash, J. E., and J. V. Sutcliffe, 1970: River flow forecasting through conceptual models Part I-A discussion of principles. J. Hydrol., 10, 282-290, https://doi.org/10.1016/ 0022-1694(70)90255-6.
Ohmura, A., 2001: Physical basis for the temperature-based meltindex method. J. Appl. Meteor., 40, 753-761, https://doi.org/ 10.1175/1520-0450(2001)040<0753:PBFTTB > 2.0.CO;2.

Pathiraja, S., H. Moradkhani, L. Marshall, A. Sharma, and G. Geenens, 2018: Data driven model uncertainty estimation in data assimilation. Water Resour. Res., 54, 1077-1092, https://doi.org/ 10.1002/2018WR022627.

Schaefli, B., B. Hingray, and A. Musy, 2007: Climate change and hydropower production in the Swiss Alps: Quantification of potential impacts and related modelling uncertainties. Hydrol. Earth Syst. Sci., 11, 1191-1205, https://doi.org/10.5194/hess-11-1191-2007.

Seidou, O., V. Fortin, A. St-Hilaire, A.-C. Favre, S. E. Adlouni, and B. Bobée, 2006: Estimating the snow water equivalent on the Gatineau catchment using hierarchical Bayesian modelling. $H y$ drol. Processes, 20, 839-855, https://doi.org/10.1002/hyp.6127.

Slater, A. G., and M. P. Clark, 2006: Snow data assimilation via an ensemble Kalman filter. J. Hydrometeor., 7, 478-493, https:// doi.org/10.1175/JHM505.1.

Stone, M., 1974: Cross-validatory choice and assessment of statistical predictions. J. Roy. Stat. Soc., 36B, 111-147.

Thiboult, A., and F. Anctil, 2015: On the difficulty to optimally implement the ensemble Kalman filter: An experiment based on many hydrological models and catchments. J. Hydrol., 529, 1147-1160, https://doi.org/10.1016/j.jhydrol.2015.09.036.

Turcotte, R., L.-G. Fortin, V. Fortin, J.-P. Fortin, and J.-P. Villeneuve, 2007: Operational analysis of the spatial distribution and the temporal evolution of the snowpack water equivalent in southern Québec, Canada. Hydrol. Res., 38, 211234, https://doi.org/10.2166/nh.2007.009.

Uboldi, F., C. Lussana, and M. Salvati, 2008: Three-dimensional spatial interpolation of surface meteorological observations from high-resolution local networks. Meteor. Appl., 15, 331345, https://doi.org/10.1002/met.76.

Zhang, H., H.-J. Hendricks Franssen, X. Han, J. A. Vrugt, and H. Vereecken, 2017: State and parameter estimation of two land surface models using the ensemble Kalman filter and the particle filter. Hydrol. Earth Syst. Sci., 21, 4927-4958, https:// doi.org/10.5194/hess-21-4927-2017. 